\title{
CONTROL DE PARÁMETROS OPERATIVOS PARA OPTIMIZAR EL FUNCIONAMIENTO DE LA RED PRINCIPAL DE TRANSPORTE Y DE DISTRIBUCIÓN DE GAS NATURAL SECO DEL PROYECTO CAMISEA I
}

\author{
THE CONTROL OF OPERATIONAL PARAMETERS TO GET AN \\ EFFICIENT PERFORMANCE OF THE MAIN NET OF \\ TRANSPORT AND DISTRIBUTION OF DRY NATURAL GAS OF \\ CAMISEA I PROJECT
}

\author{
Víctor Alfredo Aybar Chávez ${ }^{1}$
}

\begin{abstract}
RESUMEN
Los sistemas actuales de transporte y distribución de gas natural seco correspondientes al proyecto Camisea I, mueven millones de pies cúbicos diarios de este hidrocarburo; su capacidad y confiabilidad operativa repercuten en el consumidor final. La operación o el funcionamiento adecuado de ellos permiten obtener un aprovechamiento eficiente de este recurso. El trabajo tiene como objetivo demostrar que, a través del control sobre determinados parámetros operativos (específicamente, valores de presión y flujos de consumo de gas en puntos clave del sistema), es posible obtener un funcionamiento eficiente de la red principal (representado por el consumo de combustible de las estaciones de compresión). Todo ello, garantizando el libre acceso de los usuarios y cumpliendo con las condiciones contractuales entre los miembros que conforman la cadena de valor del gas.
\end{abstract}

Palabras clave.- Gas natural, Transporte, Distribución, Optimización, Modelo, Costos mínimos, Presión, Flujo.

\begin{abstract}
Current piping systems for transportation and distribution of dry natural gas in the Camisea I, project, involve the movement of millions of cubic feet of natural gas daily. Their capacity and operational reliability affect the final consumers. Their adequate operation and performance allows obtaining an efficient use of this resource. The objective of this work is to demonstrate that, through the control of certain operational parameters (specifically, values of pressure and flow of gas consumption in key points of the system), it's possible to get an efficient performance of the net (represented by users fuel consumption of the compressor stations). All of this, assumes free access of the users, and complying with contractual conditions among the members that conform the value chain of gas market.
\end{abstract}

Key words.- Natural gas, Transportation, Distribution, Optimization, Model, Minimum costs, Pressure, Flux.

\section{INTRODUCCIÓN}

De las fuentes de gas natural que existen en nuestro país, la que se halla en Camisea se constituye como la mayor esperanza energética del Perú, pues, debido a su magnitud permite y permitirá ahorrar otros tipos de combustible, al mismo tiempo que favorecerá a la creación de industrias paralelas,

\footnotetext{
${ }^{1}$ Ms, Ingeniero Mecánico-Electricista de la Universidad Nacional de Ingeniería. Supervisor de la Gerencia de
} Fiscalización de Gas natural de OSINERGMIN. 
como la petroquímica. Asimismo, provee energía limpia y barata en el corto y mediano plazo, de manera que se logre revertir el déficit en la balanza comercial de hidrocarburos, al sustituir paulatinamente las importaciones por las exportaciones. El uso racional de este energético es un tema de vital importancia para el desarrollo de nuestro país.

Este trabajo tiene como objeto demostrar que es posible controlar ciertas condiciones operativas de la red principal de los sistemas de transporte y distribución de gas natural del proyecto Camisea I, de manera que se obtenga un funcionamiento óptimo en conjunto.

\section{PROBLEMÁTICA}

En nuestro país, el control del flujo y presiones a través de los gasoductos se realiza de forma no coordinada, esto es, los transportistas y distribuidores se dedican a entregar gas enfocados en minimizar sus gastos operativos (consumo de combustible de sus estaciones de compresión, anulando la posibilidad de realizar venteos operativos) de forma independiente. En trabajos de investigación desarrollados en otros países [1, 2], para redes de gas ficticias y complejas, se ha concluido que es posible reducir al mínimo los costos de consumo de combustible de las estaciones de compresión si se considera su funcionamiento integrado.

Estos hechos ponen de manifiesto que la actual operación que se practica en la red principal puede volverse eficiente y que, por tanto, resulta relevante optimizarla, toda vez que existen herramientas que permiten ello (basados en el control de parámetros operativos). En ese contexto, es que la presente investigación se desarrolló en el ámbito de dicha red acoplada (transporte más distribución).

\section{MODELO MATEMÁTICO}

Para describir las ecuaciones a utilizar, tomemos como referencia el gráfico mostrado en la Figura 1. En dicha figura, sean $i, j$ un par de nodos que limitan los extremos de un ducto, $N$ el total de nodos presentes en la red (el $n$ de la figura sería el n-ésimo nodo), $A$ el conjunto de arcos en la red, $A_{p}$ el conjunto de arcos ductos y $A_{c}$ el conjunto de arcos donde existen compresores $\left(A=A_{p} \mathrm{U} A_{c}\right)$.

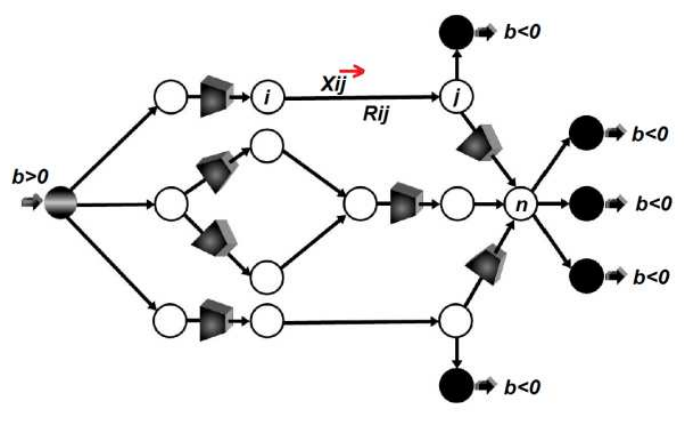

Fig. 1 Configuración de red referencial.

El balance de flujo de masa en cada nodo se calcula mediante la diferencia entre la cantidad que sale y la que entra, resultando así el flujo nodal neto dado por $b_{i}, i \in N$.

Un valor positivo (negativo) de $b_{i}$ indica que el nodo $i$ es fuente (demanda), si $b_{i}$ es igual a cero quiere decir que se trata de un nodo de paso. Una red balanceada cumple con $\sum_{i \in N} b_{i}=0$. Así, se tiene la siguiente ecuación para los flujos másicos $x_{i j}$ :

$$
\sum_{\{j \mid(i, j) \in A\}} x_{i j}-\sum_{\{j \mid(j, i) \in A\}} x_{i i}=b_{i}, i \in N
$$

El flujo de gas natural a través de cada ducto es una restricción de igualdad no lineal, la cual representa la relación entre las presiones $(p)$ en sus extremos y el flujo.

Esta ecuación es la relación más importante determinada por Osiadacz [3], y es válida para gases de alta presión, como en este caso:

$$
p_{i}^{2}-p_{j}^{2}=C_{i j} x_{i j}^{2},(i, j) \in A_{p}
$$

En esta relación, $C_{i j}$ es la resistencia del ducto, la cual se representa por la siguiente ecuación:

$$
C_{i j}=\frac{K Z S_{g} T f L}{d^{5}}
$$

Donde $K$ es una constante adimensional que toma el valor de $1,3305 \times 10^{5}$ (para el sistema métrico inglés), $Z$ es el factor de compresibilidad, $S_{g}$ es la densidad relativa del gas, $T$ es la temperatura absoluta del fluido, $f$ es el factor de fricción del tubo, $L$ es la longitud y $d$ es el diámetro interior del ducto. 
Por el lado de las estaciones de compresión, sus dominios de operación están definidos por un espacio $\left(q_{i j}, h_{i j}, s_{i j}\right)$, entre dos nodos $i j$ que conforman un arco compresor, basado en las variables de decisión que el operador de la red conoce directamente, a saber [4]:

$q_{i j}$ : Tasa interna del flujo volumétrico en el compresor $(i, j) ;(i, j) \in A_{c}$

$h_{i j}$ : Cabeza adiabática del compresor $(i, j) ;(i, j) \in$ $A_{c}$

$s_{i j}$ : Velocidad del compresor $(i, j) ;(i, j) \in A_{c}$

El dominio de operación factible de un compresor, denotado por $D^{u n i t}(i, j)$, es dado por las siguientes desigualdades:

$$
\begin{aligned}
& {S_{i j}{ }^{L}} \leq S_{i j} \leq S_{i j}{ }^{U} \\
& {R_{i j}{ }^{L}} \leq \frac{q_{i j}}{s_{i j}} \leq R_{i j}{ }^{U}
\end{aligned}
$$

A su vez, $h_{i j}, q_{i j}$ y $s_{i j}$ se relacionan de la siguiente forma:

$$
\frac{h_{i j}}{s_{i j}^{2}}=A_{H}+B_{H}\left(\frac{q_{i j}}{s_{i j}}\right)+C_{H}\left(\frac{q_{i j}}{s_{i j}}\right)^{2}+D_{H}\left(\frac{q_{i j}}{s_{i j}}\right)^{3}
$$

Donde $A_{H}, B_{H}, C_{H}$ y $D_{H}$ son coeficientes que se asumen conocidos, los cuales dependen del tipo de compresor [5] y se estiman típicamente por el método de mínimos cuadrados. $S_{i j}{ }^{L}$ y $S_{i j}{ }^{U}$ son parámetros conocidos que representan la velocidad mínima y máxima del compresor $(i, j)$, respectivamente. $R_{i j}{ }^{L}$ y $R_{i j}{ }^{U}$ representan la razón mínima y máxima de $q_{i j}{ }^{L} / s_{i j}{ }^{L}$ (surge) y $q_{i j}{ }^{U} / s_{i j}{ }^{U}$ (stonewall) para el compresor $(i, j)$, respectivamente. $Q_{i j}{ }^{L}$ y $Q_{i j}{ }^{U}$ son parámetros que indican las cotas mínimas y máximas de la tasa de flujo del compresor $(i, j)$, respectivamente. Para cada $q_{i j}$ en este rango, $h_{i j}$ es acotada inferiormente tanto por $S_{i j}{ }^{L}$ como por $R_{i j}{ }^{U}$, y acotada superiormente por $S_{i j}{ }^{U}$ y $R_{i j}{ }^{L}$.

En un análisis realizado desde la perspectiva de la optimización de redes, se llegó a la determinación de que es preferible trabajar en un espacio de operación factible definido por las variables de decisión $\left(x_{i j}, p_{i}, p_{j}\right)$, ya que el flujo másico $x_{i j}$ se conserva en cada nodo. Por lo tanto, la relación del dominio definido por $\left(q_{i j}, h_{i j}, s_{i j}\right)$ y el dominio $D^{u n i t}{ }_{(i, j)}$ en el espacio $\left(x_{i j}, p_{i}, p_{j}\right)$ se obtiene de un mapeo representado por las siguientes ecuaciones:

$$
\begin{gathered}
h_{i j}=\frac{Z R T_{S}}{m}\left[\left(\frac{p_{j}}{p_{i}}\right)^{m}-1\right] \\
q_{i j}=Z R T_{S} \frac{x_{i j}}{p_{i}}
\end{gathered}
$$

Donde los siguientes parámetros se asumen conocidos:

$T_{s}$ : Temperatura del gas natural

$Z$ : Factor de compresibilidad del gas natural

$R$ : Constante del gas natural

$m$ : Coeficiente específico de calor $(m=k-1 / k ; k=$ 1,297).

Sea:

$$
\alpha=\frac{\beta}{m} \text {, donde } \beta=Z R T_{S}
$$

Para acotar el dominio de operación factible se obtienen las siguientes restricciones $[6,7]$ :

$$
\begin{aligned}
& \alpha\left[\left(\frac{p_{j}}{p_{i}}\right)^{m}-1\right] \geq\left(S_{i j}^{L}\right)^{2} \phi\left(\frac{\beta \frac{x_{i j}}{p_{i}}}{S_{i j}^{L}}\right) \\
& \alpha\left[\left(\frac{p_{j}}{p_{i}}\right)^{m}-1\right] \leq\left(S_{i j}^{U}\right)^{2} \phi\left(\frac{\beta \frac{x_{i j}}{p_{i}}}{S_{i j}^{U}}\right) \\
& \alpha\left[\left(\frac{p_{j}}{p_{i}}\right)^{m}-1\right] \geq\left(S_{i j}^{L}\right)^{2} \phi\left(R_{i j}^{L}\right) \\
& \alpha\left[\left(\frac{p_{j}}{p_{i}}\right)^{m}-1\right] \leq\left(S_{i j}^{U}\right)^{2} \phi\left(R_{i j}^{U}\right)
\end{aligned}
$$

En virtud de ello, el modelo que representa el dominio de operación de una estación compresora es: 
$\mathrm{D}^{\prime}(\mathrm{i}, \mathrm{j})=\left\{\left(x_{i j}, p_{i}, p_{j}\right): p_{i}^{L} \leq p_{i} \leq p_{i}^{U}\right.$, donde (10), (11), (12) y (13) se cumplen $\}$

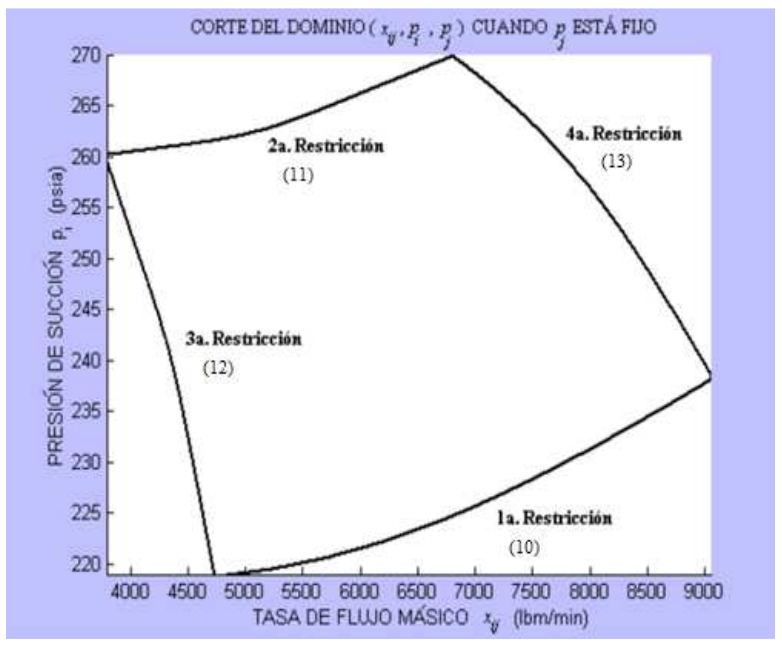

Fig. 2 Frontera de operación factible D' definido por (10), (11), (12) y (13).

La función de consumo y que se busca minimizar es la considerada en [4], la cual puede definirse en base al espacio $\left(x_{i j}, p_{i}, p_{j}\right)$ como sigue:

$$
g_{(i, j)}\left(x_{i j}, p_{i}, p_{j}\right)=\frac{c x_{i j}\left[\left(\frac{p_{j}}{p_{i}}\right)^{m}-1\right]}{\eta}
$$

Con:

$$
c=\frac{\alpha Z R T_{s}}{m}
$$

Donde $\alpha$ es una constante positiva, la cual por simplicidad se supone igual a 1 , ya que el gas que se está manejando es de alta presión.

La eficiencia adiabática es representada por $\eta, \mathrm{y}$ está dada por la siguiente ecuación:

$$
\eta=A_{E}+B_{E}\left(\frac{q_{i j}}{s_{i j}}\right)+C_{E}\left(\frac{q_{i j}}{s_{i j}}\right)^{2}+D_{E}\left(\frac{q_{i j}}{s_{i j}}\right)^{3}
$$

Donde $A_{E}, B_{E}, C_{E}$ y $D_{E}$ son coeficientes que dependen del tipo de compresor [5], y son estimados por el método de mínimos cuadrados.

\section{Elementos del modelo}

De los estudios realizados por Villalobos, Borraz y Ríos-Mercado, podemos plantear las restricciones asociadas a la operación de una red de gasoductos.

Para ello, primero se enumeran los elementos que conforman el modelo de una red de gasoductos:

Conjunto de nodos $(\mathbf{N})$, dividido a su vez en tres subconjuntos.- nodos fuente $\left(N_{f}\right.$ - centros de suministro donde se inyecta el gas al sistema), nodos demanda $\left(N_{d}\right.$ - puntos donde se requiere el gas) y nodos de paso ( $N_{p}$ - donde no se suministra ni consume gas.

Conjunto de arcos en la red (A), dividido en dos subconjuntos.- $\operatorname{arcos}$ ductos $\left(A_{p}-\operatorname{arcos}\right.$ por donde se transporta el gas) y arcos compresores $\left(A_{c}\right.$ arcos donde se encuentra una estación compresora).

Límites de presión en cada nodo.- presión mínima en el nodo $i\left(p_{i}^{L}\right)$, presión máxima en el nodo $i$ $\left(p_{i}^{U}\right)$.

Flujo neto en cada nodo $\left(\mathbf{b}_{\mathbf{i}}\right)$ - cantidad de flujo que se inyecta en los nodos suministro $\left(b_{i}>0\right.$ si $\left.i \in \mathrm{N}_{f}\right)$ $\mathrm{y}$ cantidad de flujo que se requiere en los nodos de demanda $\left(b_{i}<0\right.$ si $\left.i \in \mathrm{N}_{d}\right)$.

Propiedades físicas de cada ducto.- constante del ducto $(C)$, factor de fricción $(f)$, longitud del ducto $(L)$ y diámetro interior $(d)$.

Propiedades físicas del gas natural.- factor de compresibilidad $(Z)$, gravedad específica $\left(S_{g}\right)$, temperatura promedio $(T)$ y la constante del gas $(R)$.

\section{Restricciones}

Las limitaciones que se imponen a los valores que pueden tomar los parámetros operativos de la red se enumeran en las siguientes restricciones:

Capacidad del flujo de gas en cada ducto $\left(\mathbf{U}_{\mathrm{ij}}\right)$.flujo máximo que puede pasar por cada ducto, el cual depende de sus características geométricas.

$0 \leq x_{i j} \leq U_{i j},(i, j) \in A_{p}$

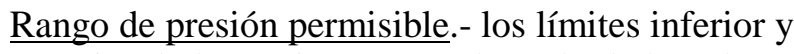
superior de la presión para cada nodo de la red son establecidos en los contratos entre los concesionarios y el Estado y/o los consumidores.

La restricción que los define se muestra a continuación: 


$$
p_{i}^{L} \leq p_{i} \leq p_{i}^{U}, i \in N
$$

Límites de operación en cada compresor.- Estos límites definen el dominio en el cual el compresor puede operar en la industria, y ya fueron descritos anteriormente:

$$
\left(x_{i j}, p_{i}, p_{j}\right) \in D_{(i, j)}^{\prime},(i, j) \in A_{c}
$$

\section{Suposiciones}

Para resolver el problema de minimización de consumo de combustible se asume lo siguiente:

- El modelo de flujo es unidimensional.

- El problema está en estado estable.

- La red está balanceada.

- Se considera un sistema isotérmico.

- La dirección de los flujos se conoce de antemano, al tratarse de una red tipo árbol.

- En cada estación compresora se establece un número fijo de unidades compresoras.

- Las unidades compresoras que se manejan en cada estación son centrífugas, idénticas e instaladas en paralelo.

\section{MARCO NORMATIVO}

Las leyes, normas, reglamentos y/o contratos que rigen el funcionamiento de los sistemas de transporte y distribución de gas natural en nuestro país, y que se consideran para la elaboración del modelo, son las siguientes:

- Ley de promoción del desarrollo de la industria del gas natural, y su reglamento

- Reglamento de transporte de hidrocarburos por ductos

- Reglamento de distribución de gas natural por red de ductos

- Contratos BOOT (build, own, operate and transfer) de transporte y distribución de gas natural seco

- Contratos de prestación de servicio entre los agentes que conforman la cadena de valor del gas

\section{MÉTODO DE SOLUCIÓN}

Los componentes básicos del método de solución pueden describirse de manera general como sigue:
Programa computacional a utilizar.- Se utiliza el GAMS 23.9.2.

Pre-procesamiento.- Técnica que tiene dos objetivos fundamentales: Refinar las cotas del dominio de operación factible de las variables, y reducir el tamaño de la instancia de red sin alterar su estructura matemática.

Asignación de flujos.- Dada la topología del sistema de transporte y distribución que abordamos, es posible obtener un conjunto de flujos factibles iniciales, de manera eficiente, en base en una técnica de asignación "hacia atrás".

Solución óptima de las variables de presión.- Se encuentra un conjunto de presiones óptimas aplicando una técnica de programación dinámica no secuencial (para el conjunto de flujos factibles obtenido en la fase anterior). Bajo los fundamentos teóricos encontrados en Borraz (donde se demuestra que en topologías no cíclicas existe una única manera de asignar los flujos), en esta fase obtenemos soluciones óptimas globales.

\section{APLICACIÓN EN EL SISTEMA OBJETIVO}

La red principal de gas natural está conformada por dos grandes sistemas: la red de transporte (cuyo propietario es Transportadora de gas del Perú - TGP) y la red de distribución en Lima y Callao (cuyo propietario es Gas Natural de Lima y Callao - Cálidda). Ver Fig. 3

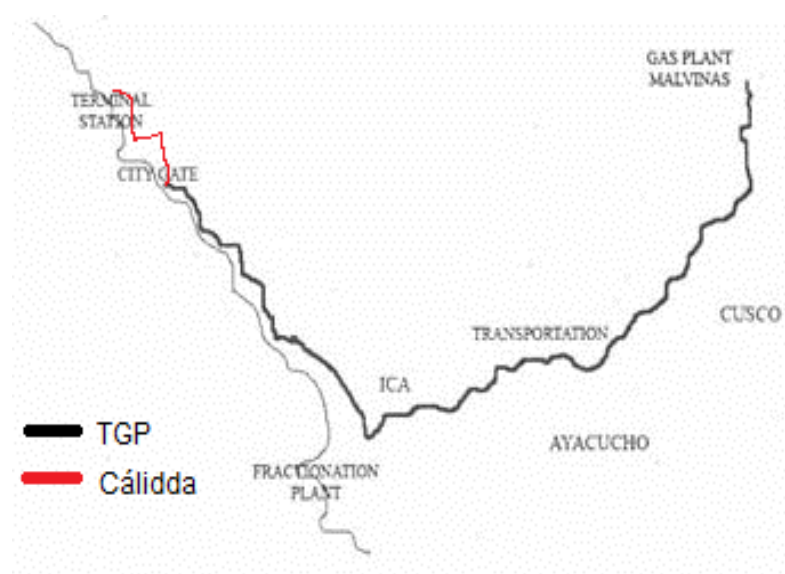

Fig. 3 Esquema del sistema de transporte de TGP y Cálidda.

Los componentes de ambos sistemas, vía red principal, son los siguientes. Ver Tabla 1 y 2 : 
Tabla 1. Componentes del sistema de transporte.

\begin{tabular}{|c|c|c|c|}
\hline $\begin{array}{c}\text { Nodo } \\
\mathbf{N}^{\mathbf{o}}\end{array}$ & Tipo de componente & $\begin{array}{c}\text { Ubicación aproximada, en } \\
\text { progresivas kilométricas }\end{array}$ & Principales características \\
\hline 1 & Inicio de ducto de transporte & 0,0 & Ubicado en Malvinas \\
\hline 2 & Tramo selva & $\begin{array}{l}\text { Inicio: } 0,0 \\
\text { Fin: } 211,0\end{array}$ & Ducto de acero de $\phi 32 ”$ \\
\hline $2-3$ & Estación de compresión & 211,0 & $\begin{array}{l}4 \text { compresores marca Dresser-Rand, } \\
\text { modelo D10R3S }\end{array}$ \\
\hline $3-5$ & Tramo sierra & $\begin{array}{l}\text { Inicio: } 211,0 \\
\text { Fin: } 519,0\end{array}$ & Ducto de acero de $\phi 24 "$ \\
\hline $5-10$ & Tramo costa & $\begin{array}{l}\text { Inicio: } 519,0 \\
\text { Fin: } 729,0\end{array}$ & Ducto de acero de $\phi 18^{\prime \prime}$ \\
\hline $\begin{array}{c}27-30- \\
29\end{array}$ & Loop costa & $\begin{array}{l}\text { Inicio: } 595,0 \\
\text { Fin: } 699,0\end{array}$ & Ducto de acero de $\phi 24 "$ \\
\hline $2-31$ & $\begin{array}{l}\text { Ducto hacia Pampa } \\
\text { Melchorita }\end{array}$ & Inicio: 210,5 & $\begin{array}{l}\text { Ducto de acero de } \phi 34 " ; \text { long. aprox. } \\
408,1 \mathrm{~km}\end{array}$ \\
\hline $5-32$ & $\begin{array}{l}\text { Ducto hacia planta de } \\
\text { fraccionamiento }\end{array}$ & Inicio: 519,5 & $\begin{array}{l}\text { Ducto de acero de } \phi 8 " ; \text { long. aprox. } \\
40,4 \mathrm{~km} \text {. }\end{array}$ \\
\hline 9 & $\begin{array}{c}\text { Derivación EGESUR - } \\
\text { EGASA }\end{array}$ & 528,6 & Longitud despreciable \\
\hline 6 & Derivación DUKE ENERGY & 699,7 & Longitud despreciable \\
\hline 7 & Derivación ENERSUR & 700,0 & Longitud despreciable \\
\hline 8 & Derivación KALLPA & 701,0 & Longitud despreciable \\
\hline
\end{tabular}


Tabla 2. Componentes del sistema de distribución.

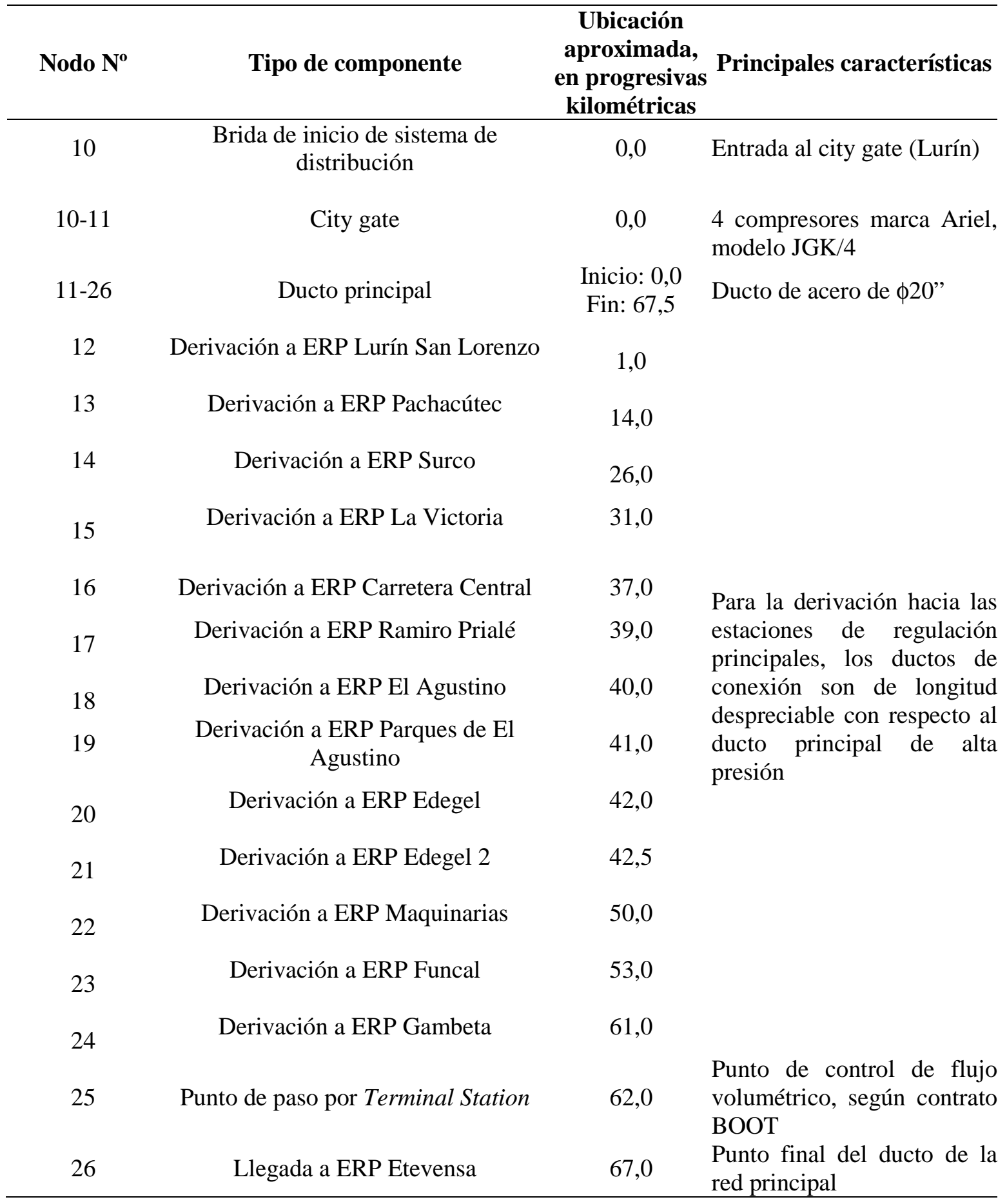

La configuración de nodos, tuberías y estaciones topología-, utilizada para la simulación, lo podemos ver en la Figura 4, (los círculos representan los nodos): 


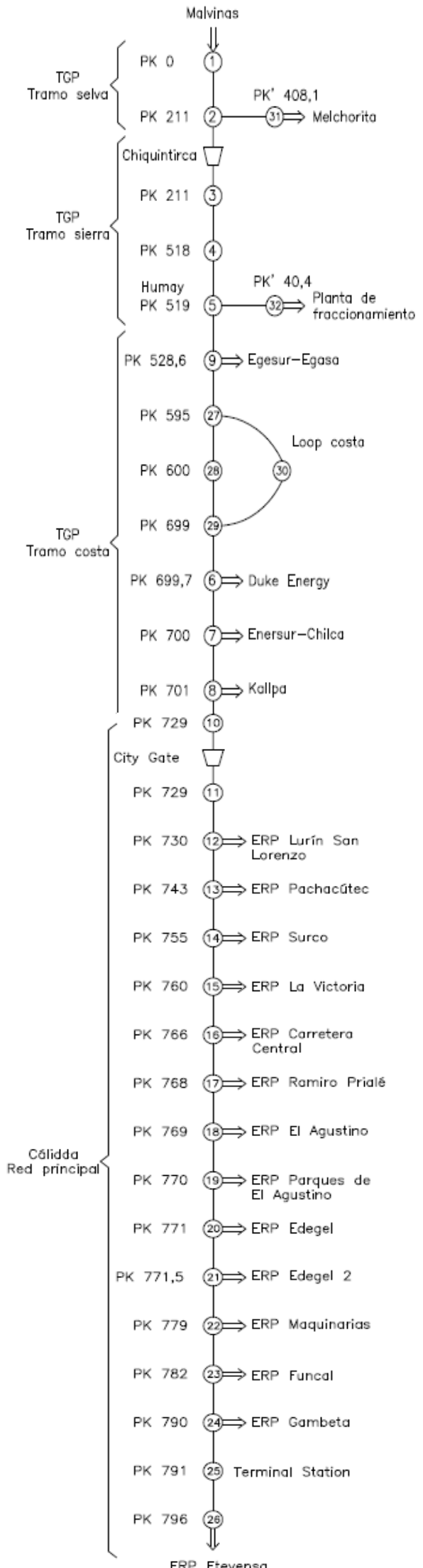

Fig. 4 Topología de la red a modelar. 


\section{Condiciones de contorno}

Estas condiciones están definidas por las capacidades del flujo de gas en cada ducto, el rango de presión permisible y los límites de operación en cada compresor. Ver Tabla 3 y 4.

Tabla 3. Capacidad del flujo de gas en cada ducto del sistema a modelar.

\begin{tabular}{cccccc}
\hline Arco & $\begin{array}{c}\text { Capacidad } \\
\text { (MMsPCD) }\end{array}$ & Arco & $\begin{array}{c}\text { Capacidad } \\
\text { (MMsPCD) }\end{array}$ & Arco & $\begin{array}{c}\text { Capacidad } \\
\text { (MMsPCD) }\end{array}$ \\
\hline $1-2$ & 1150 & $30-29$ & 1150 & $16-17$ & 255 \\
$2-31$ & 650 & $29-6$ & 1150 & $17-18$ & 255 \\
$3-4$ & 1150 & $6-7$ & 1150 & $18-19$ & 255 \\
$4-5$ & 1150 & $7-8$ & 1150 & $19-20$ & 255 \\
$5-32$ & 1150 & $8-10$ & 1150 & $20-21$ & 255 \\
$5-9$ & 1150 & $11-12$ & 255 & $21-22$ & 255 \\
$9-27$ & 1150 & $12-13$ & 255 & $22-23$ & 255 \\
$27-28$ & 1150 & $13-14$ & 255 & $23-24$ & 255 \\
$28-29$ & 1150 & $14-15$ & 255 & $24-25$ & 255 \\
$27-30$ & 1150 & $15-16$ & 255 & $25-26$ & 255 \\
\hline
\end{tabular}

Tabla 4. Límites de presión máximo y mínimo en cada nodo del sistema a modelar (en bar-a).

\begin{tabular}{cccccc}
\hline Nodo & $\begin{array}{c}\text { Presión } \\
\text { máxima }\end{array}$ & $\begin{array}{c}\text { Presión } \\
\text { mínima }\end{array}$ & Nodo & $\begin{array}{c}\text { Presión } \\
\text { máxima }\end{array}$ & Presión mínima \\
\hline 1 a 9 & 147 & 40 & 21 & 50 & 32 \\
10 & 120 & 40 & 22 & 50 & 20 \\
11 & 50 & 32 & 23 & 50 & 6 \\
12 & 50 & 6 & 24 & 50 & 11 \\
13 & 50 & 11 & 25 & 50 & 32 \\
14 & 50 & 11 & 26 & 50 & 32 \\
15 & 50 & 20 & 27 & 147 & 40 \\
16 & 50 & 11 & 28 & 147 & 40 \\
17 & 50 & 11 & 29 & 147 & 40 \\
18 & 50 & 11 & 30 & 147 & 40 \\
19 & 50 & 6 & 31 & 147 & 81 \\
20 & 50 & 32 & 32 & 147 & 70 \\
\hline
\end{tabular}

\section{Condiciones iniciales}

Como datos de entrada para el modelo a implementar consideramos a los flujos volumétricos demandados por los consumidores o ERP's presentes en la red. Para poder aplicar el esquema numérico en el sistema deseado, se consideraron demandas reales correspondientes a diez (10) días operativos. Dichos parámetros operativos están disponibles en las páginas web de los concesionarios y de OSINERGMIN $[8,9,10]$.

A modo de ejemplo, se muestra a continuación los escenarios de tres días en la Tabla 5. 
Tabla 5. Flujos volumétricos considerados en tres escenarios, para proceder con la simulación del modelo numérico.

\begin{tabular}{|c|c|c|c|c|}
\hline \multirow[b]{2}{*}{ Nodo } & \multirow[b]{2}{*}{ Tipo } & \multicolumn{3}{|c|}{ Flujo neto (MMsPCD) } \\
\hline & & $\begin{array}{c}\text { Escenario del } \\
26 / 11 / 2012\end{array}$ & $\begin{array}{c}\text { Escenario del } \\
01 / 11 / 2012\end{array}$ & Escenario del 15/10/2012 \\
\hline 1 & Oferta & 1042,86 & 988,25 & 1018,72 \\
\hline 2 & Paso & 0.00 & 0.00 & 0.00 \\
\hline 3 & Paso & 0.00 & 0.00 & 0.00 \\
\hline 4 & Paso & 0.00 & 0.00 & 0.00 \\
\hline 5 & Paso & 0.00 & 0.00 & 0.00 \\
\hline 6 & Demanda & 21,28 & 0.00 & 28,92 \\
\hline 7 & Demanda & 115,41 & 95,55 & 56,36 \\
\hline 8 & Demanda & 75,82 & 52,59 & 120,28 \\
\hline 9 & Demanda & 10,65 & 13,64 & 11,54 \\
\hline 10 & Paso & 0.00 & 0.00 & 0.00 \\
\hline 11 & Paso & 0.00 & 0.00 & 0.00 \\
\hline 12 & Demanda & 7,79 & 11,86 & 8,09 \\
\hline 13 & Demanda & 7,79 & 11,86 & 8,09 \\
\hline 14 & Demanda & 7,79 & 11,86 & 8,09 \\
\hline 15 & Demanda & 7,79 & 11,86 & 8,09 \\
\hline 16 & Demanda & 7,79 & 11,86 & 8,09 \\
\hline 17 & Demanda & 7,79 & 11,86 & 8,09 \\
\hline 18 & Demanda & 10,06 & 9,06 & 7,62 \\
\hline 19 & Demanda & 7,79 & 11,86 & 8,09 \\
\hline 20 & Demanda & 11,61 & 0.00 & 0.00 \\
\hline 21 & Demanda & 14,23 & 0.00 & 0.00 \\
\hline 22 & Demanda & 19,91 & 24,37 & 20,13 \\
\hline 23 & Demanda & 7,79 & 11,86 & 8,09 \\
\hline 24 & Demanda & 7,79 & 11,86 & 8,09 \\
\hline 25 & Paso & 0.00 & 0.00 & 0.00 \\
\hline 26 & Demanda & 69,23 & 58,21 & 74,12 \\
\hline 27 & Paso & 0.00 & 0.00 & 0.00 \\
\hline 28 & Paso & 0.00 & 0.00 & 0.00 \\
\hline 29 & Paso & 0.00 & 0.00 & 0.00 \\
\hline 30 & Paso & 0.00 & 0.00 & 0.00 \\
\hline 31 & Demanda & 609,99 & 609,99 & 609,99 \\
\hline 32 & Demanda & 14,56 & 18,1 & 16,95 \\
\hline
\end{tabular}

Los valores anteriores se mantendrán constantes en la simulación, asumiendo el cálculo para estado estable.

Otras variables que requieren de un valor inicial para poder efectuar las iteraciones dentro del cálculo, son:
Para cada nodo.- Presión.

Para las estaciones de compresión.- Cabeza adiabática, velocidad de rotación, relación entre el flujo volumétrico y la velocidad, capacidad de transporte de gas en la estación, eficiencia, y costo de consumo de combustible. 
Los valores iniciales para estas variables será el promedio aritmético de los límites superior e inferior que puedan tener.

Este valor se irá recalculando a medida que se ejecutan las iteraciones del modelo.

\section{RESULTADOS}

\section{Aplicación en el sistema optimizado}

A continuación se muestran los resultados obtenidos para la topología objetivo, en la condición de sistema optimizado.

Tabla 6. Consumo de combustible mínimo por arco compresor y total, en $W$.

\begin{tabular}{ccccc}
\hline Escenario & $\mathbf{N}^{\circ}$ iteraciones & $\begin{array}{c}\text { Arco } \\
\text { compresor 2.3 }\end{array}$ & $\begin{array}{c}\text { Arco } \\
\text { compresor } \\
\mathbf{1 0 . 1 1}\end{array}$ & Consumo total \\
\hline $26 / 11 / 2012$ & 40 & $9,574.0$ & $5,426.6$ & $15,000.7$ \\
$01 / 11 / 2012$ & 23 & $8,327.4$ & $5,470.0$ & $13,797.4$ \\
$15 / 10 / 2012$ & 29 & $9,043.2$ & $5,162.2$ & $14,205.4$ \\
$01 / 10 / 2012$ & 23 & $8,185.4$ & $5,241.6$ & $13,427.0$ \\
$15 / 09 / 2012$ & 34 & $12,039.4$ & $17,068.6$ & $29,108.0$ \\
$01 / 09 / 2012$ & 28 & $9,090.8$ & $14,930.6$ & $24,021.4$ \\
$15 / 08 / 2012$ & 35 & $9,553.6$ & $12,991.6$ & $22,545.2$ \\
$01 / 08 / 2012$ & 37 & $11,565.1$ & $15,284.4$ & $26,849.5$ \\
$15 / 07 / 2012$ & 27 & $9,166.2$ & $9,107.1$ & $18,273.3$ \\
$01 / 07 / 2012$ & 27 & $10,691.0$ & $5,891.7$ & $16,582.7$ \\
\hline
\end{tabular}

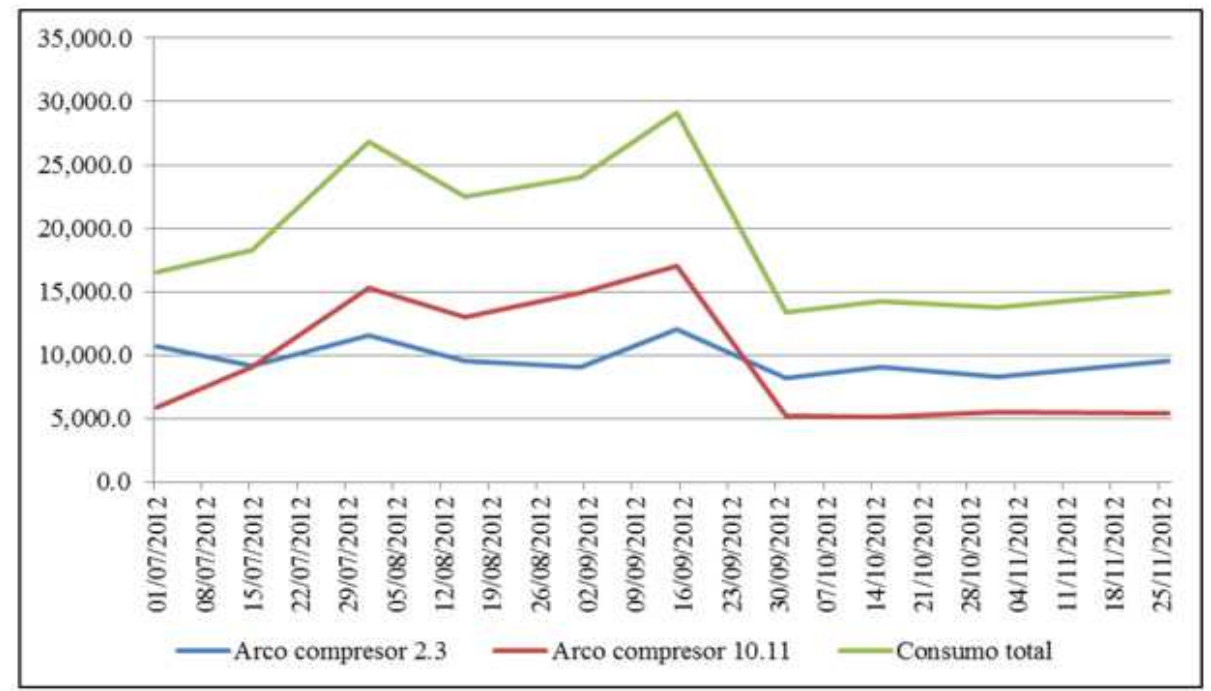

Fig. 5 Gráfico de consumo de combustible (en W) a través de los escenarios.

En la Tabla 6 y Figura 5, se puede observar que el consumo mínimo es relativamente estable en el tiempo para el ducto de transporte, y con más variaciones en el de distribución, siendo éste finalmente quien determina la variación del consumo total a través del tiempo. 
Tabla 7. Eficiencia de cada arco compresor, en \%.

\begin{tabular}{ccc}
\hline Escenario & Arco compresor 2.3 & Arco compresor 10.11 \\
\hline $26 / 11 / 2012$ & 80.8 & 73.4 \\
$01 / 11 / 2012$ & 81.2 & 73.9 \\
$15 / 10 / 2012$ & 80.8 & 70.0 \\
$01 / 10 / 2012$ & 81.7 & 71.1 \\
$15 / 09 / 2012$ & 85.2 & 61.1 \\
$01 / 09 / 2012$ & 82.5 & 61.0 \\
$15 / 08 / 2012$ & 81.4 & 61.0 \\
$01 / 08 / 2012$ & 85.2 & 61.1 \\
$15 / 07 / 2012$ & 82.6 & 61.1 \\
$01 / 07 / 2012$ & 84.0 & 77.6 \\
\hline
\end{tabular}

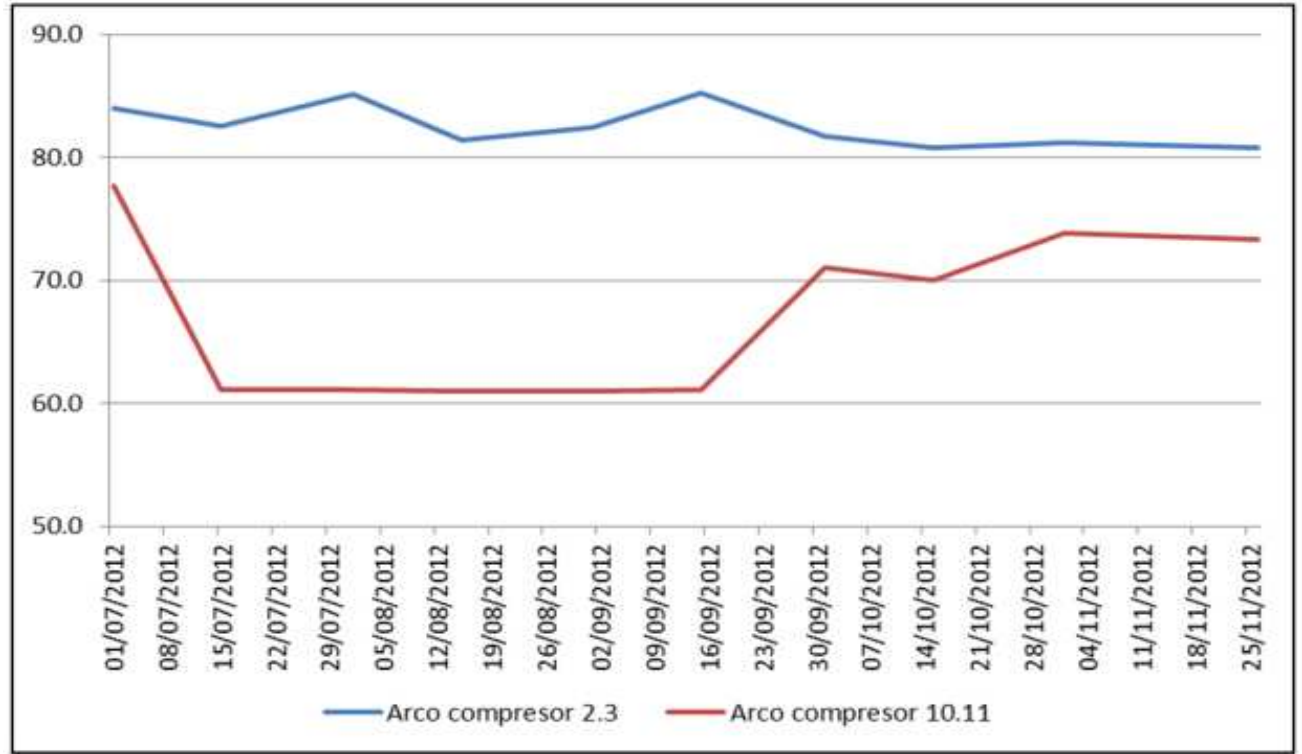

Fig. 6 Gráfico de variación de la eficiencia (en \%) para las estaciones de compresión. en estado de optimización.

En la Tabla 7 y Figura 6, se puede deducir que la estación de transporte trabaja con una eficiencia mayor a la de distribución. Si esto lo relacionamos con el consumo de combustible que tienen las estaciones, se puede afirmar que el consumo de la estación de distribución está en el orden de la estación de transporte debido a que es menos eficiente que ésta. 


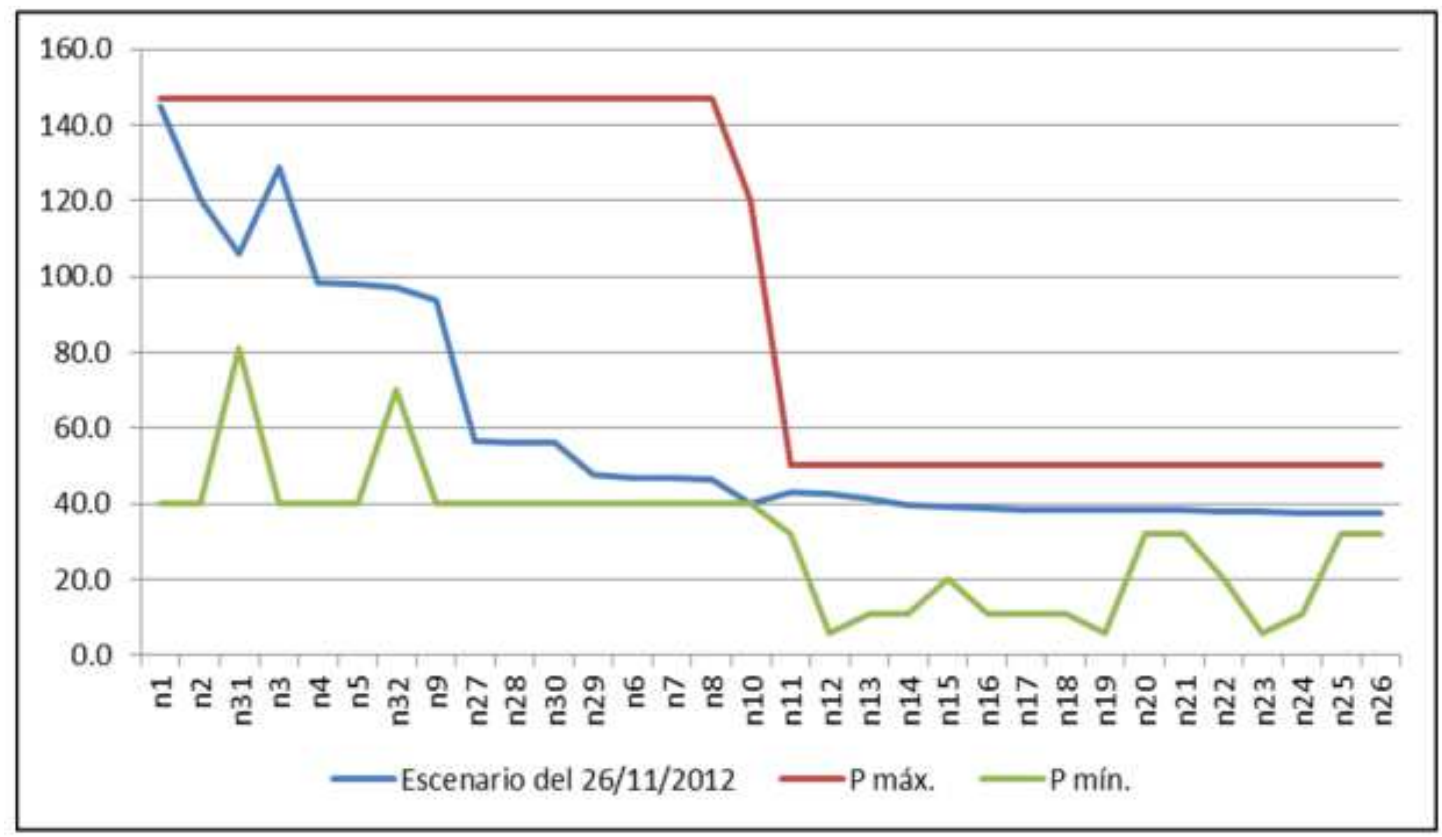

Fig. 7 Gráfico de la variación de presión a lo largo de todos los nodos (en bar-a), para el escenario del 26/11/2012.

De la Fig. 7, la cual mantiene la tendencia a través de los diez escenarios simulados, se deduce que el nodo crítico dentro de la operación global de la red principal está alrededor del número 10-11, lo que corresponde al city gate de Lurín. Dentro de los resultados del sistema optimizado, se encontró que en varios de los escenarios, la presión en el nodo 10 se sitúa en 40 bar-a, cuando la presión permitida normativamente debe de estar comprendida en el rango de 40 a 120 bar-a.

\section{Aplicación en el sistema no optimizado}

Un sistema no optimizado significa que no trabaja en condiciones óptimas de asignación de presiones en los nodos de la red, con el mismo supuesto de requerimientos volumétricos (en condiciones estándar) en los puntos de demanda. Con la finalidad de contar con presiones reales de referencia, se utilizaron los reportes operativos del transportista y distribuidor disponibles en las páginas web indicadas en $[8,9$ y 10$]$, tomando como datos aquellas presiones correspondientes a los nodos $\mathrm{N}^{\circ} 1,5$ y 11 (Malvinas, Humay y salida del City Gate, respectivamente).
Con estas presiones y los flujos másicos como dato, se pasó a calcular aguas abajo o aguas arriba, según convenga, los valores de presión de los demás nodos, utilizando para ello la ecuación de flujo de gas a través de ductos; una vez teniendo definidos los flujos y presiones, y de la mano con la información sobre el dominio de operación de los compresores, se procedió a calcular el costo asociado a su funcionamiento. Los resultados para este sistema se muestran en la Tabla 8.

\section{Comparación y análisis entre el sistema optimizado y el no optimizado}

Una vez obtenidos los resultados, podemos apreciar que, para iguales valores de flujos y demás características físicas de los componentes de la red, existen diferencias en los valores de presión (que cumplen con la normativa vigente) y de consumo de combustible total para todo el sistema.

Las diferencias en consumo de combustible se resumen a continuación: 
Tabla 8. Resumen de resultados del consumo de combustible total entre el sistema optimizado y el no optimizado (en W).

\begin{tabular}{cccc}
\hline Escenario & $\begin{array}{c}\text { Sistema } \\
\text { optimizado }\end{array}$ & $\begin{array}{c}\text { Sistema no } \\
\text { optimizado }\end{array}$ & Diferencia relativa \\
\hline $26 / 11 / 2012$ & $15,000.7$ & $39,718.0$ & $62.23 \%$ \\
$01 / 11 / 2012$ & $13,797.4$ & $31,755.2$ & $56.55 \%$ \\
$15 / 10 / 2012$ & $14,205.4$ & $33,387.7$ & $57.45 \%$ \\
$01 / 10 / 2012$ & $13,427.0$ & $27,990.1$ & $52.03 \%$ \\
$15 / 09 / 2012$ & $29,108.0$ & $38,520.6$ & $24.44 \%$ \\
$01 / 09 / 2012$ & $24,021.4$ & $25,462.6$ & $5.66 \%$ \\
$15 / 08 / 2012$ & $22,545.2$ & $36,013.7$ & $37.40 \%$ \\
$01 / 08 / 2012$ & $26,849.5$ & $34,974.6$ & $23.23 \%$ \\
$15 / 07 / 2012$ & $18,273.3$ & $24,823.7$ & $26.39 \%$ \\
$01 / 07 / 2012$ & $16,582.7$ & $34,747.9$ & $52.28 \%$ \\
\hline
\end{tabular}

De esta Tabla, se observa que la diferencia entre ambos sistemas se da para todos los escenarios, con diferencias relativas de considerable magnitud, las cuales están dentro de un promedio de 39,7\%. Esto quiere decir que esta herramienta demuestra que es posible controlar los valores de presiones, dentro de los rangos establecidos técnica y normativamente, para distintos escenarios de demanda, que reduzcan al mínimo el consumo total de combustible de las estaciones compresoras existentes en la red y anulando el venteo operativo, procurando así su funcionamiento eficiente. Ello, siempre y cuando se considere al transporte y distribución de gas natural como un todo, un sistema global.

\section{CONCLUSIONES}

Mediante el modelo elaborado, se demostró que a través del control adecuado de parámetros operativos (presiones y volúmenes demandados) se optimiza el funcionamiento de la red principal de transporte y de distribución de gas natural seco del proyecto Camisea I, como resultado de la minimización del consumo de combustible de las estaciones compresoras en conjunto.
Utilizando los softwares proporcionados por los fabricantes de los comprensores, se obtuvieron determinados parámetros operativos que permitieron estimar a través del método de mínimos cuadrados los coeficientes de las ecuaciones que relacionan la eficiencia, la cabeza adiabática, el caudal volumétrico y la velocidad de rotación de estos equipos.

Dentro de la topología modelada no se consideró ningún punto de demanda asociado a los venteos operativos, por lo que el sistema optimizado es factible sin la necesidad de efectuar estos venteos.

Es necesario incluir las condiciones de entrega del gas natural al transportista como parte del proceso de optimización, ya que la presión en el punto de recepción influye directamente en el consumo de combustible del sistema de compresión del ducto de transporte.

El city gate de Lurín se constituye como una zona crítica dentro de la red, toda vez que sus parámetros operativos fluctúan cerca del límite establecido por las normas aplicables. 


\section{AGRADECIMIENTOS}

A los docentes de Postgrado FIM y a los revisores de este trabajo, por haberse dado tiempo dentro de las innumerables actividades académicas $y$ profesionales a que se dedican, para brindarme parte sus invalorables conocimientos en la estructuración del trabajo. Asimismo, a Omar por haberme brindado las facilidades para la obtención de la neurálgica información que se necesitó para la aplicación del modelo desarrollado en este trabajo.

\section{REFERENCIAS}

1. R. Ríos-Mercado, D. Cobos-Zaleta, "A MINLP Model for a Minimizing Fuel Consumption on Natural Gas Pipeline Networks". Artículo técnico. Memorias del XI Congreso Latino Iberoamericano de Investigación de Operaciones, Chile, 2002.

2. R. Ríos-Mercado, S. Kim, E. Boyd, "Efficient Operation of Natural Gas Transmission Systems: A Network-Based Heuristic for Cyclic Structures". Artículo técnico. Texas, Institute of Information.

3. Osiadacz, J., "Simulation and Analysis of
Gas Networks". Artículo técnico. Gulf Publishing Company, EE.UU, 1987.

4. Borraz, Conrado, "Una metodología de solución basada en programación dinámica no secuencial y búsqueda tabú para la operación eficiente de sistemas de transporte de gas natural en estado estable". Tesis de maestría. Universidad Autónoma de Nueva León, México, 2004.

5. http://turbocalc.dresser-rand.com/

6. Villalobos Morales, J., "Preprocesamiento de un problema de optimización de redes de gas natural". Tesis doctoral. Universidad Autónoma de Nueva León, México, 2002.

7. Flores Villarreal, H., "Operación eficiente de sistemas de transporte de gas natural mediante el método de gradiente reducido generalizado". Tesis de maestría. Universidad Autónoma de Nueva León, México, 2005.

8. http://www.calidda.com.pe/

9. http://gasnatural.osinerg.gob.pe/contenidos/ empresas_sector/

10. http://www.tgp.com.pe/resumen/Default.aspx

Correspondencia: vic_aach@yahoo.es

Recepción de originales: setiembre 2014

Aceptación de originales: octubre 2014 